\title{
Successful electrode insertion for spinal cord stimulation after balloon adhesiolysis in a patient with epidural adhesion - A case report -
}

Received October 16, 2020

Revised February 2, 2021

Accepted February 21, 2021

\section{Dong-Min Hyun, Chan-Hye Park, Yujin Kim, and Seong-Soo Choi}

Department of Anesthesiology and Pain Medicine, Asan Medical Center, University of Ulsan College of Medicine, Seoul, Korea

\begin{abstract}
Background: Spinal cord stimulation (SCS) can be successfully performed using highly developed implantation techniques. However, anatomical barriers, such as epidural adhesion, may impede placing the electrode for SCS in an adequate position.

Case: A 60-year-old female who had SCS with an electrode at the T9-10 level removed because she had a wound infection at the back incision site. After the wound infection was completely resolved, we tried to re-insert the SCS electrode. However, it was difficult to advance it up to the T11 level due to epidural adhesion. We performed a combined epidural adhesiolysis using balloon decompression with an inflatable balloon catheter. After that, the SCS lead was successfully placed up to the T11 level, and implantation of SCS was performed.
\end{abstract}

Conclusions: When a patient has epidural adhesion, an epidural adhesiolysis with an inflatable balloon catheter may help the insertion of the SCS electrode in the epidural space.

Keywords: Balloon; Infection; Percutaneous epidural adhesiolysis; Spinal cord stimulation.
Neuromodulation approaches targeting the spinal cord, dorsal root ganglia, and peripheral nerves have been used in the treatment of chronic pain syndromes with traditionally neuropathic pathologies [1]. However, more recently, neuromodulation has been used even in mixed pathologies with nociceptive pain, including failed back surgery syndrome and complex regional pain syndrome (CRPS) $[2,3]$. Among these, CRPS is characterized by pain, in combination with sensory, autonomic, trophic and motor abnormalities [4]. Patients often develop severe functional disability, depression, and social isolation as a consequence of CRPS [5]. Although there are many treatment modalities for CRPS, patient satisfaction remains poor. Hence, patients with CRPS are often candidates for spinal cord stimulation (SCS).

The SCS system could be easily implanted due to highly developed implantation techniques, and the rate of procedure failure is only $2.3 \%$ of the total failed SCS rate [6]. There are only few descriptions of anatomical barriers that prevent the adequate placement of spinal cord stimulation

This is an Open Access article distributed under the terms of the Creative Commons Attribution Non-Commercial License (http://creativecommons.org/licenses/by-nc/4.0) which permits unrestricted non-commercial use, distribution, and reproduction in any medium, provided the original work is properly cited.

Copyright (C) the Korean Society of Anesthesiologists, 2021 
[7]. Sometimes, patients suspected of spinal stenosis or anatomical anomalies such as epidural adhesion or fibrosis face difficulties in the correct placement of the SCS electrode, such that it is often requires magnetic resonance imaging (MRI) evaluation. There are many possible causes of epidural adhesion, including surgical trauma, annular tear, infection, hematoma, or intrathecal contrast material [8]. In addition, previous studies showed that the combined epidural balloon decompression and adhesiolysis with an inflatable balloon catheter could expand the epidural space by mechanical adhesiolysis $[9,10]$ with the rare complications including dura matter puncture (3.3\%), subdural injection (1.8\%), vascular injection (1.5\%), disc injection $(2.2 \%)$, and hypotension during balloon adhesiolysis (1.5\%). Therefore, percutaneous epidural balloon adhesiolysis without surgical laminectomy may overcome the difficulty of SCS electrode implantation caused by epidural adhesion.

We herein present a case report of a successful re-implantation of an SCS electrode in a female who had epidural adhesion after the removal of a previous SCS electrode. After using an inflatable balloon catheter for decompression and adhesiolysis of the epidural space, we could successfully reinsert the SCS lead into the target site.

\section{CASE REPORT}

We obtained written informed consent from the patient after surgery to publish this report.

A 60-year-old female presented with chronic pain in the right ankle and foot. She had right foot ligament tear, which included anterior talofibular ligament, calcaneoufibular ligament and posterior talofibular ligament, followed by a modified Brostrom surgery on the right foot in March 2017. However, the patient still felt a painful sensation, the numerical rating scale (NRS) was 10 of 10, at the right foot. She also complained of allodynia, burning and tingling sensation, leg edema, and change of skin color with a dystonic posture of the right foot. The result of electromyography and nerve conduction velocity was no abnormal findings. However, bone scan showed diffusely decreased perfusion, blood pool and bone uptake of right foot and quantitative sudomotor axon reflex test suggest sympathetic postganglionic sudomotor dysfunction or sweat gland abnormality in right distal leg. Department of orthopedic surgery in our institution gave an opinion that there were no joint pathology, bony abnormality and nothing to cause the pain with orthopedic opinions. Following a clinical assessment, she was diagnosed with CRPS type 1 according to the International Association for the Study of Pain clinical diagnostic criteria [11]. Although she was treated with several medications, physical therapy, lumbar epidural blocks, and lumbar sympathetic ganglion blocks, her symptoms remained intractable.

In August 2018, she was considered for SCS trial. During the SCS trial, strict aseptic techniques and prophylactic antibiotics (cefazolin) were used. A guide needle for an electrode was inserted through the right L1-2 paramedian epidural space, and the tip of the electrode was placed at the T9-10 level. After the induced paresthesia was confirmed at her painful sites, the lead was fixed. During a test period, her pain and allodynia decreased from 9 to 0 on NRS. We implanted a permanent implantable pulse generator (IPG) in a subcutaneous pocket of her left lower abdomen.

On the fifth postoperative day, the patient had back wound dehiscence and pus discharge back incision site. The neurological examination was normal. Computed tomography (CT) and thoracic MRI showed no abnormality except a back skin wound. The culture report of the specimen of the back wound discharge revealed extended-spectrum $\beta$-lactamase (ESBL) producing Escherichia coli. Following a discussion with the Department of Infectious Disease in our institution, ertapenem was immediately started and the patient underwent surgical wound exploration under general anesthesia. The wound problem was resolved; hence, she was discharged on ciprofloxacin and followed-up at the out-patient clinic. However, wound dehiscence at the back incision site was observed again after 2 months. Although a plastic surgeon performed wound revision with local flap twice, the wound problem did not resolve, so we decided to remove the SCS system.

In March 2019, we consulted the Department of Infectious Disease in our institution and found that there was no evidence of residual infection. The patient was treated through oral medication, but complained the same symptom as before. Therefore, we decided to re-insert the SCS electrode. The electrode insertion was planned through the contralateral T12-L1 epidural space to evade the previous infection site. However, during the lead insertion, it was impossible to advance up to the T11 level of the epidural space. Moreover, the contrast dye did not spread above the T11 level of the epidural space. Consequently, we concluded that the epidural adhesion was interrupting the electrode advance. We supposed that epidural adhesiolysis to 
resolve the epidural adhesion would be helpful for the insertion of the SCS. After epidural adhesiolysis with a Racz catheter, however, the electrode was still not placed up to the T11 level (Fig. 1). Therefore, we additionally tried to perform the combined epidural balloon decompression and adhesiolysis with an inflatable balloon catheter (Zineu ${ }^{\circledR}$, JUVENUI, Korea) (Fig. 2A, B). After combined balloon decompression and adhesiolysis, the lead moved well,

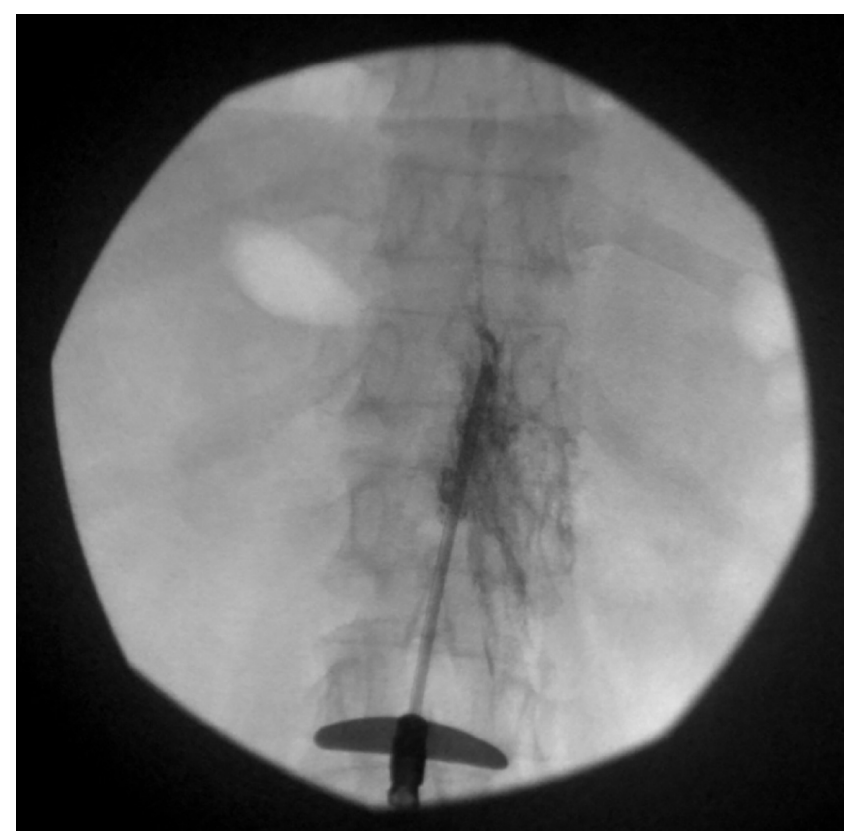

Fig. 1. Contrast dye was not spread above the T11-12 epidural space under fluoroscopic view.

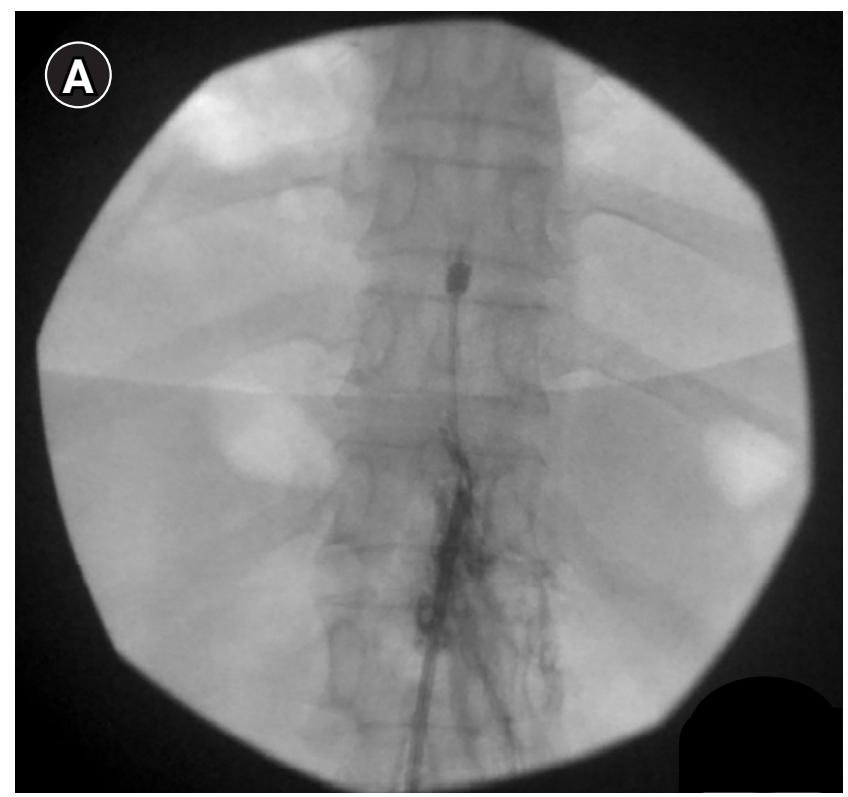

and we successfully implanted the SCS electrode at the T910 level. After the induced paresthesia was confirmed at her painful sites, the new IPG device was then repositioned in a subcutaneous pocket, on the left lower abdominal region (Fig. 3). Tingling sense decreased from 10 to 2 on NRS, and burning sensation decreased from 10 to 5 on NRS. The intravenous administration of ertapenem to cover ESBL producing $E$. Coli was continued for a 2 week period, following which the blood culture result was negative. There were no postoperative complications, and the patient was discharged in a good physical state. The symptom of patient has been well controlled with the SCS to date.

\section{DISCUSSION}

CRPS is a complex biopsychosocial condition which responds most often to integrated multidisciplinary treatment which includes psychological, medical, and physical and occupational therapies [12]. A 2013 Cochrane review reported low quality evidence for the pharmacologic treatment of CRPS with bisphosphonates, calcitonin, and subanesthetic intravenous ketamine, physical and occupational therapy, sympathetic ganglion blockade [13]. After failure of the above approaches, many clinicians suggested a trial of SCS. Permanent implantation is usually pursued following a successful trial, with an emphasis on functional improvement and normalization of activities of daily living. In the present case, treatments with several medications,

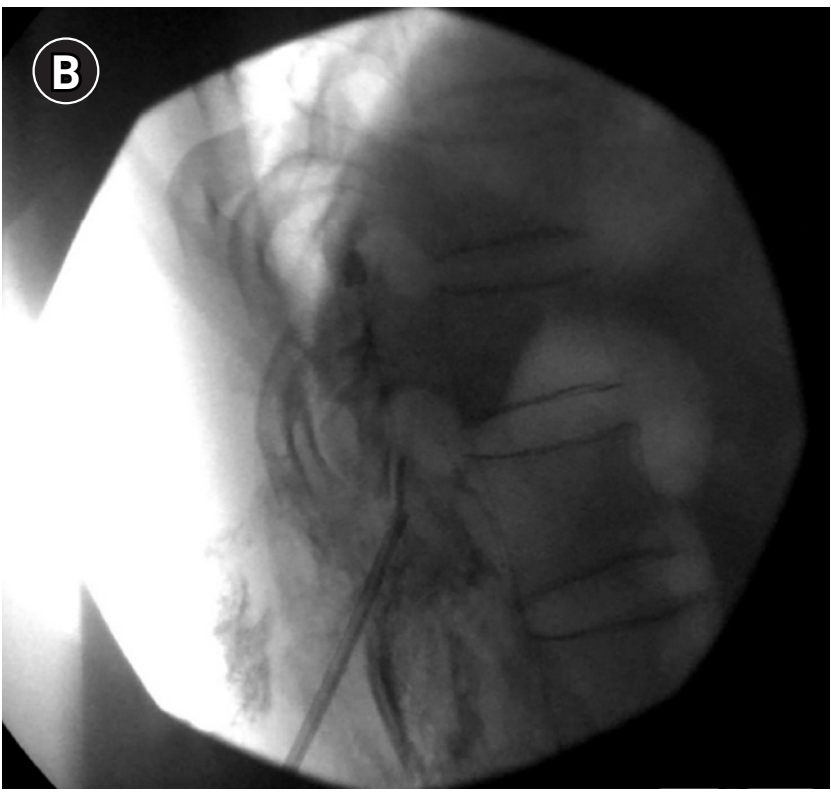

Fig. 2. Fluoroscopic view showing the position of inflatable balloon catheter in the T11 epidural space. The balloon filled with contrast medium are shown at T10-11 level. (A) Fluoroscopic anteroposterior view, (B) Fluoroscopic lateral view. 


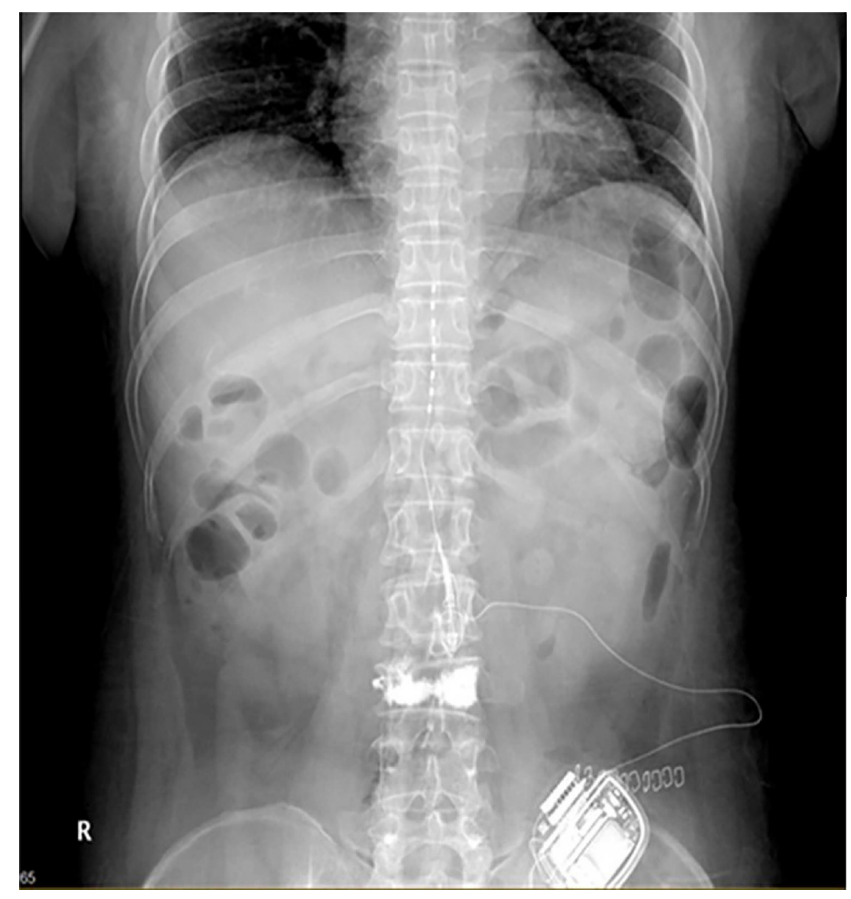

Fig. 3. Postoperative X-ray (anteroposterior view) of a round type lead placed at the T9-10 level, connected to a rechargeable implantable pulse generator. The electrode was placed through the contralateral L1-2 epidural space to evade the previous infection site.

repeated physical therapy, epidural steroid injections, lumbar sympathetic ganglion blocks or other spinal interventional procedures did not yield effective pain control or functional improvements. However, SCS implantation led to effective pain relief and improvements in the patient's functional status. Therefore, despite the initial removal of the SCS system due to wound infection, we re-inserted the SCS lead after resolution of the infection. According to a multicenter retrospective study, SCS infection rate was $2.45 \%$ [14]. Localized incisional pain and wound erythema were the most common presenting signs. The most common causative organism was Staphylococcus aureus and the IPG pocket was the most common site of the SCS-related infection [14]. In the present case, only wound infection, but not epidural space infection was found on CT and MRI images. Therefore, we determined that patient's epidural adhesions due to previous SCS lead placement prevented the electrode from moving up to the T11 level epidural space. In this situation, surgical laminectomy and adhesiolysis can be considered for the correct placement of the SCS electrode. Given that these adhesion can be effectively treated with percutaneous epidural adhesiolysis [15] and serious complications may be more frequent in surgical laminectomy (hematoma, 5.20\%; renal, $2.80 \%$; pulmonary,
$1.60 \%$; cardiac, $1.0 \%$ ) [16] than in percutaneous epidural adhesiolysis (epidural abscess $1.0 \%$; respiratory depression, $0.5 \%$ ) [17], we tried the percutaneous epidural adhesiolysis on our patient. However, we failed to place the SCS electrode up to the target site after percutaneous adhesiolysis with the RACZ catheter. Inflatable balloon catheters could be more effective in adhesiolysis than balloon-less catheters, because inflatable balloon catheters provide mechanical adhesiolysis as well as chemical adhesiolysis, while balloon-less catheters provide chemical adhesiolysis only [18]. In addition, the inflatable balloon catheter can more effectively remove or relieve severe degrees of adhesion [19]. Therefore, we decided to perform epidural adhesiolysis and decompression using an inflatable balloon catheter. As shown in Fig. 1, there is an inflatable balloon at the tip of the catheter to remove adhesions or relieve stenosis. Ballooning with a contrast agent helps in visually verifying the degree of adhesion or stenosis by the extent of distortion of the balloon. Conventionally, when SCS insertion using percutaneous technique fails, the only choice is SCS insertion using a surgical technique. However, this could increase the rate for complications of SCS [20]. Percutaneous SCS electrode positioning was successfully performed after percutaneous epidural adhesiolysis with the inflatable balloon catheter avoiding surgical laminectomy in the present patient.

In conclusion, we report the first case describing the use of an inflatable balloon catheter for epidural adhesiolysis in a patient with CRPS via epidural adhesion requiring SCS electrode implantation. Our case suggests that the inflatable balloon catheter should be considered for performing epidural adhesiolysis when SCS leads fail to be inserted due to epidural adhesion before determining surgical laminectomy for electrode placement.

\section{CONFLICTS OF INTEREST}

No potential conflict of interest relevant to this article was reported.

\section{DATA AVAILABILITY STATEMENT}

The datasets generated during and/or analyzed during the current study are available from the corresponding author on reasonable request. 


\section{AUTHOR CONTRIBUTIONS}

Conceptualization: Seong-Soo Choi. Data curation: Yujin Kim. Writing - original draft: Dong-Min Hyun, Chan-Hye Park. Writing - review \& editing: Dong-Min Hyun, SeongSoo Choi. Supervision: Seong-Soo Choi.

\section{ORCID}

Dong-Min Hyun, https://orcid.org/0000-0002-3735-7383 Chan-Hye Park, https://orcid.org/0000-0003-3838-4268 Yujin Kim, https://orcid.org/0000-0001-9212-3224 Seong-Soo Choi, https://orcid.org/0000-0002-2333-0235

\section{REFERENCES}

1. Aló KM, Holsheimer J. New trends in neuromodulation for the management of neuropathic pain. Neurosurgery 2002; 50: 690703. discussion 703-4.

2. Kemler MA, Barendse GA, van Kleef M, de Vet HC, Rijks CP, Furnée CA, et al. Spinal cord stimulation in patients with chronic reflex sympathetic dystrophy. N Engl J Med 2000; 343: $618-24$.

3. Kapural L, Yu C, Doust MW, Gliner BE, Vallejo R, Sitzman BT, et al. Novel 10-kHz high-frequency therapy (HF10 therapy) is superior to traditional low-frequency spinal cord stimulation for the treatment of chronic back and leg pain: the SENZA-RCT randomized controlled trial. Anesthesiology 2015; 123: 851-60.

4. Marinus J, Moseley GL, Birklein F, Baron R, Maihöfner C, Kingery WS, et al. Clinical features and pathophysiology of complex regional pain syndrome. Lancet Neurol 2011; 10: 63748.

5. Shim H, Rose J, Halle S, Shekane P. Complex regional pain syndrome: a narrative review for the practising clinician. Br J Anaesth 2019; 123: e424-33.

6. Jang HD, Kim MS, Chang CH, Kim SW, Kim OL, Kim SH. Analysis of failed spinal cord stimulation trials in the treatment of intractable chronic pain. J Korean Neurosurg Soc 2008; 43: 859.

7. Zhang Y, Wood MJ, Gilligan C. Spinal cord stimulation in a patient with spinal epidural lipomatosis. Pain Med 2011; 12: 377 81.

8. Manchikanti L, Staats PS, Singh V, Schultz DM, Vilims BD, Jasper JF, et al. Evidence-based practice guidelines for interventional techniques in the management of chronic spinal pain. Pain Physician 2003; 6: 3-81.
9. Kim SH, Choi WJ, Suh JH, Jeon SR, Hwang CJ, Koh WU, et al. Effects of transforaminal balloon treatment in patients with lumbar foraminal stenosis: a randomized, controlled, double-blind trial. Pain Physician 2013; 16: 213-24.

10. Choi SS, Lee JH, Kim D, Kim HK, Lee S, Song KJ, et al. Effectiveness and factors associated with epidural decompression and adhesiolysis using a balloon-inflatable catheter in chronic lumbar spinal stenosis: 1-year follow-up. Pain Med 2016; 17: 476-87.

11. Merskey H, Bogduk N. Classification of chronic pain: descriptions of chronic pain syndromes and definitions of pain terms. 2nd ed. Seattle, IASP Press. 1994

12. Stanton-Hicks MD, Burton AW, Bruehl SP, Carr DB, Harden RN, Hassenbusch SJ, et al. An updated interdisciplinary clinical pathway for CRPS: report of an expert panel. Pain Pract 2002; 2 : $1-16$.

13. O'Connell NE, Wand BM, McAuley J, Marston L, Moseley GL. Interventions for treating pain and disability in adults with complex regional pain syndrome. Cochrane Database Syst Rev 2013; 2013: CD009416.

14. Bendel MA, O'Brien T, Hoelzer BC, Deer TR, Pittelkow TP, Costandi S, et al. Spinal cord stimulator related infections: findings from a multicenter retrospective analysis of 2737 implants. Neuromodulation 2017; 20: 553-7.

15. Manchikanti L, Knezevic NN, Sanapati MR, Boswell MV, Kaye AD, Hirsch JA. Effectiveness of percutaneous adhesiolysis in managing chronic central lumbar spinal stenosis: a systematic review and meta-analysis. Pain Physician 2019; 22: E523-50.

16. Li G, Patil CG, Lad SP, Ho C, Tian W, Boakye M. Effects of age and comorbidities on complication rates and adverse outcomes after lumbar laminectomy in elderly patients. Spine (Phila Pa 1976) 2008; 33: 1250-5.

17. Talu GK, Erdine S. Complications of epidural neuroplasty: a retrospective evaluation. Neuromodulation 2003; 6: 237-47.

18. Karm MH, Choi SS, Kim DH, Park JY, Lee S, Park JK, et al. Percutaneous epidural adhesiolysis using inflatable balloon catheter and balloon-less catheter in central lumbar spinal stenosis with neurogenic claudication: a randomized controlled trial. Pain Physician 2018; 21: 593-606.

19. Choi SS, Joo EY, Hwang BS, Lee JH, Lee G, Suh JH, et al. A novel balloon-inflatable catheter for percutaneous epidural adhesiolysis and decompression. Korean J Pain 2014; 27: 178-85.

20. Bendersky D, Yampolsky C. Is spinal cord stimulation safe? A review of its complications. World Neurosurg 2014; 82: 135968. 